\title{
A neglected lower impacted premolar leading to cervical necrotizing faciitis
}

\begin{abstract}
Cervical necrotizing fasciitis is a rare polymicrobial infection characterized by extensive, rapidly spreading necrosis of the subcutaneous tissue and superficial fascia. This paper describes a case of necrotizing fasciitis of odontogenic origin in a 55-year-old man who presented with a dental abscess associated with an impacted lower premolar after prolonged use of removable dentures. Treatment included extensive surgical debridement, systemic antibiotics, and intensive care. This case highlights the importance of proper evaluation and diagnosis before the rehabilitation of edentulous patients. A panoramic radiograph can show the presence of an impacted tooth, which must be removed before installation of a removable denture to prevent further complications like that.
\end{abstract}

Volume 9 Issue 6 - 2018

\author{
Eduardo Morato Oliveira, Rodrigo Carvalho \\ Pinto Coelho, Maraísa Aparecida Pinto, \\ Augusto César Sette-Dias, Allyson Nogueira \\ Moreira, Evandro Guimarães Aguiar, Leandro \\ Napier Souza
}

Department of Oral and Maxillofacial Surgery, Federal University of Minas Gerais, Brazil

\begin{abstract}
Correspondence: Leandro Napier de Souza, Department of Oral and Maxillofacial Surgery, Dentistry College, Federal University of Minas Gerais, Av. Antônio Carlos, 6627 - Pampulha, Belo Horizonte, MG, 31270-90।, Brazil, Email leandronapiardesouza@gmail.com
\end{abstract}

Received: November 05, 2018 | Published: December II, 2018

\section{Introduction}

Necrotizing fasciitis (NF) is a rare, severe, and potentially fatal infection of the superficial tissues that spreads along the fascial planes, characterized by rapid, progressive and generalized necrosis of the subcutaneous tissues and fascia. ${ }^{1-3} \mathrm{NF}$ most commonly occurs in the abdomen, perineum, and extremities, and is uncommon in the cervicofacial region. When affecting head and neck region, it is usually a complication of odontogenic or peritonsillar infection, posttraumatic skin infection, osteoradionecrosis, or salivary gland infection..$^{4-7}$ Cervicofacial necrotizing fasciitis (CNF) is caused by polymicrobial or mixed aerobic-anaerobic infection, and its initial course resembles abscess or cellulitis. There is no gender or race predilection. Patients are typically febrile, tachycardic, dehydrated, and may have difficulty in swallowing due to edema or trismus. The overlying skin is often erythematous and tense. There is associated pain, swelling and crepitation due to gas formation. The causative organisms spread rapidly through the fascial planes of the head and neck. This deep infection causes vascular occlusion, ischemia, and necrosis of superficial tissues, which is the hallmark of this condition. ${ }^{5,8-11}$ Misdiagnosis and delayed treatment can result in severe systemic toxicity, carotid artery erosion, jugular vein thrombophlebitis, aspiration pneumonia, meningitis, and descending mediastinitis. The mortality rate associated with this condition ranges from $15 \%$ to $40 \%{ }^{6-13}$ Early diagnosis, aggressive surgical treatment, broad-spectrum intravenous antibiotics, airway maintenance, and adjunctive hyperbaric oxygen therapy are the keys to successful treatment of CNF. ${ }^{12-15}$ This paper reports a case of CNF due to odontogenic infection associated with an impacted tooth. A successful outcome was achieved through aggressive therapy, including radical debridement, appropriate antibiotics, and intense supportive care, followed by surgical tooth removal.

\section{Case presentation}

A 55-year-old man with a history of hypertension, alcoholism, and heavy smoking was referred to the emergency department of Odilon Behrens Metropolitan Hospital, Belo Horizonte, Brazil, with a chief complaint of diffuse swelling and tenderness in the right submandibular region. The patient reported that the signs and symptoms had started four days before. Two days after symptom onset, he saw a dentist, who diagnosed odontogenic cellulitis and prescribed oral antibiotics (amoxicillin 500mg and metronidazole $500 \mathrm{mg}$, both every 8 hours), a non-steroidal anti-inflammatory agent (diclofenac sodium $50 \mathrm{mg}$ every 8 hours), and local heat application, but with no improvement or relief of symptoms. Physical examination showed moderate, diffuse swelling of the right submandibular space, which crossed the midline to the contralateral space. Overlying skin was erythematous, tense, with a small fluctuant area on the right side. On intraoral examination, the floor of the mouth was raised. A small portion of the crown of an impacted tooth was visible on the alveolar ridge, in the right mandibular premolar area. The patient had been fully edentulous for more than 30 years, and used a removable complete denture. Additional observations included marked trismus, dysphagia, and fever $\left(38^{\circ} \mathrm{C}\right)$. A left lateral oblique radiograph showed severe alveolar bone resorption and an impacted tooth in the right premolar area of the mandible (Figure 1). Computed tomography (CT) showed involvement of the right submandibular and parapharyngeal spaces (Figure 2). A complete blood count revealed a white cell count of $12.4 \times 10^{9} / \mathrm{L}$ with $86 \%$ neutrophils, suggesting acute bacterial infection. The other vital signs were within normal limits. An initial diagnosis of Ludwig's angina secondary to dental impaction was established. Incision and drainage of the swelling, performed under local anesthesia, yielded copious amounts of foul-smelling pus, which was sent for culture and antimicrobial sensitivity studies. The patient was admitted for empiric broad-spectrum parenteral antibiotic therapy (IV clindamycin $600 \mathrm{mg}$ every 6 hours and IV gentamicin $240 \mathrm{mg}$ every 24 hours) and supportive care. The patient's condition deteriorated during the 48 hours following incision and drainage. The swelling extended to the right neck and thorax and there were severe pain, persistent fever, and increased dysphagia. The skin overlying the neck was tense and a necrotic area, which was sleeping with foul-smelling pus, had developed along the right side. This lesion extended from the submandibular area to the upper sternal region. A tracheotomy was performed. Under general anesthesia, abscess 
drainage and debridement of necrotic tissue were performed. Massive necrosis of the skin, subcutaneous fat, and fascia were evident. All necrotic tissue was debrided thoroughly and all spaces were irrigated with normal saline solution. Culture and sensitivity report showed growth of Gram-positive cocci and Gram-negative rods. Patient continued under clindamycin (600mg IV every 6 hours) and gentamicin (240mg IV every 24 hours) for 10 days. Tracheostomy was kept for 3 days after debridement, and patient was discharged from the ICU after 5 days. Wound was debrided daily for removal of small necrotic fragments, irrigated with normal saline solution, and packed with dressings soaked in 1\% silver sulfadiazine (Figure 3). Tooth was extracted after 25 days, when mouth opening was better and cervical wound had improved. After 31 days, reconstructive surgery was performed closing wound with a skin graft. The patient recovered from this procedure uneventfully with satisfactory healing of the wound (Figure 4) and was discharged from the hospital 39 days after admission.

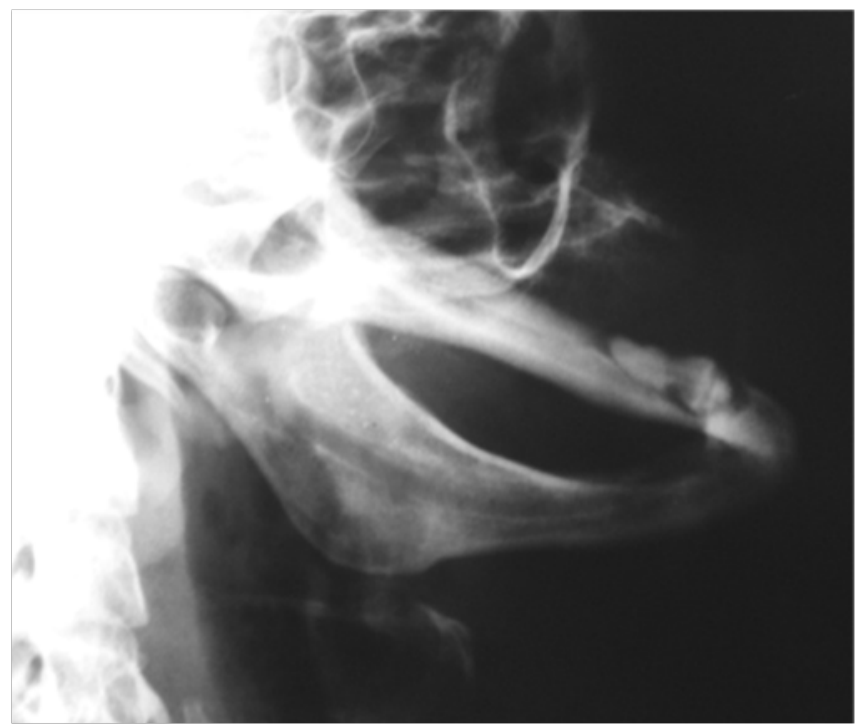

Figure I Left lateral oblique radiograph showing the presence of an impacted tooth in the mandible.

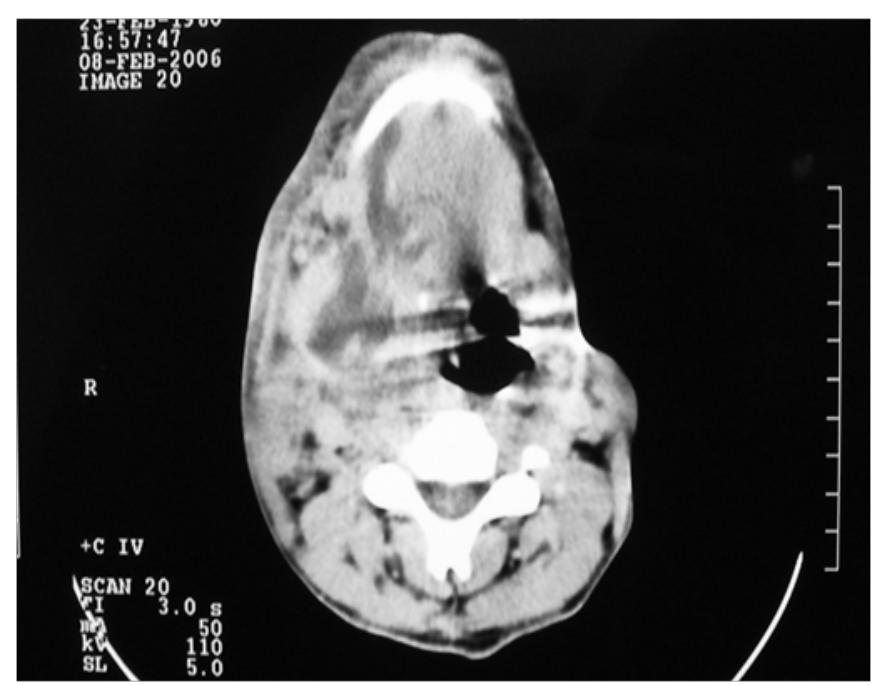

Figure 2 Axial CT image showing air collections in the submandibular and cervical regions on the right side.

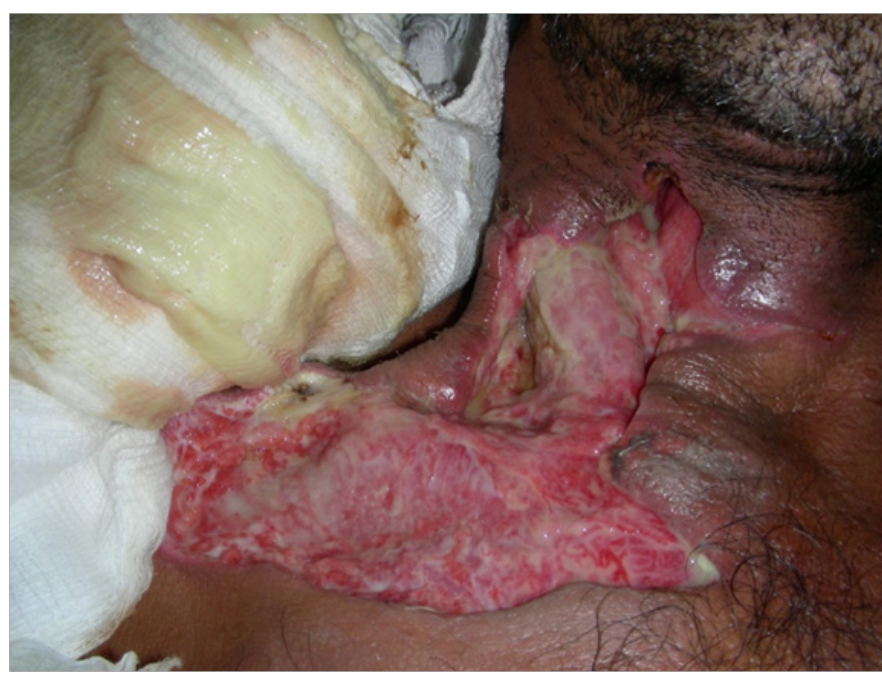

Figure 3 Surgical debridement of the affected region.

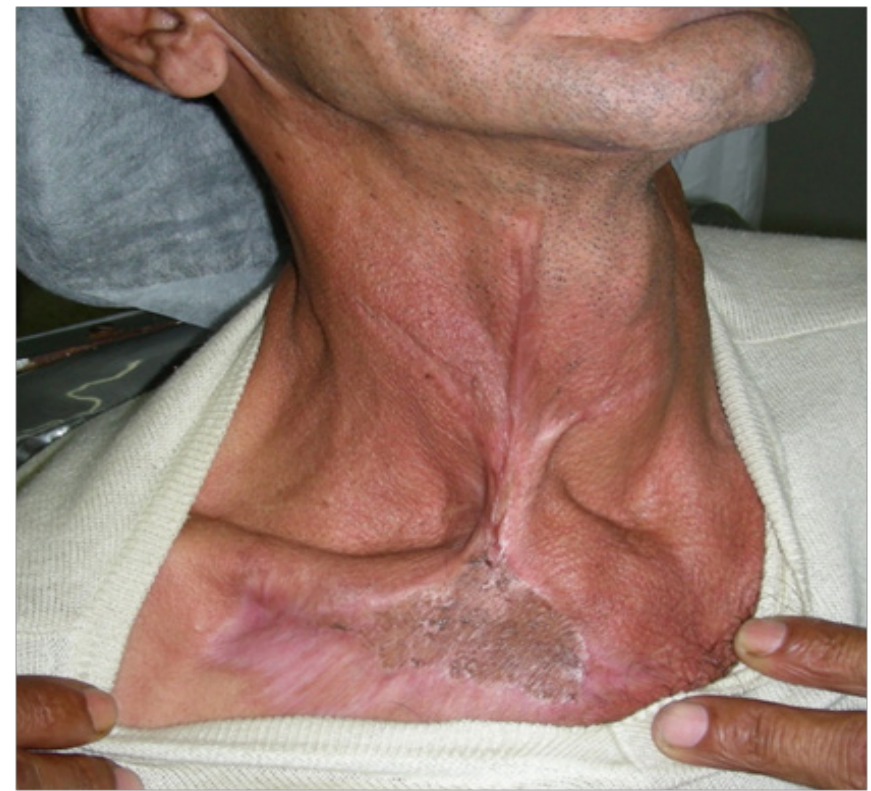

Figure 4 Complete recovery of the affected area after 38 days.

\section{Discussion}

$\mathrm{CNF}$ is a rare, rapidly progressive, life-threatening, polymicrobial infection, commonly of odontogenic origin. ${ }^{1-20}$ According to the literature, $\mathrm{CNF}$ of odontogenic origin often involves the mandibular second and third molar, as the apices of these teeth extend below the mylohyoid insertion. An infective process originating from these teeth easily traverses into the submandibular space. ${ }^{6,9}$ In the case reported herein, the mandibular second premolar was the origin. Mortality is higher in patients over 50 years of age, those with associated systemic illnesses such as diabetes mellitus or peripheral vascular disease, immunocompromised state, and when there is a delay in diagnosis. ${ }^{7-9}$ Alcoholism and tobacco abuse are also associated with $\mathrm{CNF}^{2}$ This may be due to a direct effect of these substances or to the malnutrition and poor health status that is often observed in these patients. Alcoholism alone or associated with malnutrition increases the risk of $\mathrm{CNF}$ and is observed in $22 \%$ to $45 \%$ of cases. $^{2,5,21}$ Regardless 
of comorbidities, the ability to quickly recognize when additional surgical treatment is necessary might be the key to improving patient survival. An accurate and prompt diagnosis is the most crucial aspect to decrease the morbidity and mortality rates of this devastating condition. ${ }^{2,4,521,22}$ In this case, the patient was a 55-year-old man with a history of hypertension, smoking, and alcohol consumption; he was initially treated for an odontogenic abscess, but failed to respond to drainage and double antibiotic therapy. His infection progressed to necrotizing fasciitis within 6 days, requiring second exploration and drainage under general anesthesia. The patient responded well to definitive treatment and achieved a complete recovery after 39 days of hospitalization. This successful outcome can be attributed to the early diagnosis and rapid, aggressive treatment, with wide local surgical debridement, intravenous antibiotics, fluid and electrolyte replacement, and supportive care, as recommended in the literature..$^{5-2.5}$ According to Farrier et al., ${ }^{8}$ antibiotic therapy alone is insufficient for the management of necrotizing fasciitis and surgical intervention is mandatory. The basic principles for the treatment of odontogenic abscesses are incision and drainage and source control., ${ }^{21,23,26}$ In our case, $\mathrm{x}$-ray examination showed an impacted tooth (mandibular right first premolar), but the patient had severe trismus and dysphagia, which prevented extraction of the tooth that had caused the infection. Moreover, the atrophic alveolar ridge might have fractured during extraction, causing additional morbidity. Therefore, the tooth was removed only after clinical improvement. Ideally, during routine dental care, the dentist will conduct a thorough clinical and radiographic examination covering all oral regions. In this case, a non-extracted impacted tooth was exposed in the oral cavity due to alveolar bone resorption, probably secondary to prolonged denture use. This created a route of contamination, which, combined with other factors, led to progression of infection. This reinforces the importance of obtaining a panoramic radiograph to check for impacted teeth before any attempt at prosthodontic rehabilitation. ${ }^{27}$ Imaging studies are useful in the evaluation of CNF in the early stages; however, conventional radiographs provide little information. Once the disease has progressed sufficiently, small soft-tissue opacities representing gas formation can be observed on CT, as can compromise airway. $1,2,5,7,21,22$ The availability of CT imaging has greatly enhanced early diagnosis. Treatment is often beset by complications, secondary not only to the patient's underlying medical condition but also to the delay in, or insufficiency of, surgical treatment. ${ }^{4,7,21}$ Antimicrobial agents initially used in this case were targeted toward the most frequently encountered microorganisms in an infection of odontogenic origin. The antibiotic regimen should be modified according to culture and sensitivity results. ${ }^{5,14,21}$ In life-threatening infections, when appropriate antibiotic therapy must be instituted promptly in the absence of culture findings, it is essential that the physician be aware of the microbiologic flora involved in each clinical condition. ${ }^{9} \mathrm{NF}$ is typically polymicrobial, often with anaerobic Enterobacteriaceae and occasionally with facultative anaerobes. ${ }^{1,2,26}$ Administration of inappropriate treatment may lead to selective growth of resistant population and severe complications for the patient. It is our position that, in sepsis, not only aerobes but also anaerobes are important. Due to the characteristics of the microbial flora involved in the etiology of oral infections, conventional microbiological procedures for isolation, identification, and characterization of the causative agent, when possible, are costly, and may require very specific conditions and a very long time until results are available. In such cases, if infection is life-threatening, evidence-based empiric antibiotic therapy must be instituted immediately. A suitable combination of antibiotics effective against both aerobic and anaerobic bacteria is important. We agree with other reports that clindamycin plus gentamicin appears to be efficient for initial combination therapy in such patients. ${ }^{1,16,26}$ Reconstruction is typically initiated once the patient has been stable for a number of days. ${ }^{19,25}$ In the case reported herein, complete healing of the affected regions was achieved, with less esthetic and no functional restrictions at the end of treatment. This patient had a protracted hospital stay, in part because alcohol abuse and smoking led to a difficult recovery. A review of reported cases identified mean hospital stays ranging from 15 to 50 days ${ }^{2,9,10,18}$ with most days spent in the ICU. $\cdot{ }^{25}$ Fatal outcomes can be prevented by professionals who are at the first point of patient contact, ${ }^{25}$ although the window of opportunity for surgical treatment extends to the advanced stages of the disease. ${ }^{21,25,26}$ Adjunctive therapies as hyperbaric oxygen (HBO) therapy and frozen sections biopsies facilitate the eradication of diseased tissue, and might decrease the mortality and limit the extent of debridement in patients with CNF. ${ }^{11,16}$ $\mathrm{CNF}$ is a rare, rapidly progressive, life-threatening polymicrobial infection that may be encountered by dentists, as odontogenic infection is one of its main causes. Reducing the mortality of CNF depends on early detection and adequate surgical treatment, antibiotic therapy, and intensive care. A multispecialty approach to treatment is essential to improving outcomes and decreasing morbidity and mortality rates. The correct treatment planning in edentulous patients requires orthopantomographic radiography prior to the installation of a removable denture. In the presence of impacted teeth the surgical removal must be considered to prevent any further bone resorption, which may lead to exposure of the tooth in the oral cavity leading to the possibility of subsequent infection.

\section{Acknowledgments}

None.

\section{Conflicts of interest}

The authors declare that there is no conflict of interest.

\section{References}

1. Antunes, AA, Avelar RL, de Melo WM, et al. Extensive cervical necrotizing fasciitis of odontogenic origin. $J$ Craniofac Surg. 2013;24(6):e594-597.

2. Camino Junior R, Naclerio-Homem MG, Cabral LM, et al. Cervical necrotizing fasciitis of odontogenic origin in a diabetic patient complicated by substance abuse. Braz Dent J. 2014;25(1):69-72.

3. Cruz Toro P, Callejo Castillo A, Tornero Salto J, et al. Cervical necrotizing fasciitis: report of 6 cases and review of literature," Eur Ann Otorhinolaryngol Head Neck Dis. 2014;131(6):357-359.

4. Tung-Yiu W, Jehn-Shyun H, Ching-Hung C, et al. Cervical necrotizing fasciitis of odontogenic origin: a report of 11 cases. J Oral Maxillofac Surg. 2000;58(12):1347-1352.

5. Whitesides L, Cotto-Cumba C, Myers RA. Cervical necrotizing fasciitis of odontogenic origin: a case report and review of 12 cases. J Oral Maxillofac Surg. 2000;58(2):144-151.

6. Yadav S, Verma A, Sachdeva A. Facial necrotizing fasciitis from an odontogenic infection," Oral Surg Oral Med Oral Pathol Oral Radiol. 2012;113(2):e1-4.

7. Leyva P, Herrero M., Eslava JM, et al. Cervical necrotizing fasciitis and diabetic ketoacidosis: literature review and case report. Int J Oral Maxillofac Surg. 2013;42(12):1592-1595. 
8. Farrier JN, Kittur MA, Sugar AW. Necrotising fasciitis of the submandibular region; a complication of odontogenic origin. Br Dent J. 2007;202(10):607-609.

9. Subhashraj K, Jayakumar N, Ravindran C. Cervical necrotizing fasciitis: an unusual sequel of odontogenic infection," Med Oral Patol Oral Cir Bucal. 2008;13(12):E788-791.

10. Maria A, Rajnikanth K. Cervical necrotizing fasciitis caused by dental infection: A review and case report. Natl J Maxillofac Surg. 2010;1(2):135-138.

11. Chunduri NS, Madasu K, Tammannavar PS, et al. Necrotising fasciitis of odontogenic origin. BMJ Case Rep. 2013.

12. Flanagan CE, Daramola OO, Maisel RH, et al. Surgical debridement and adjunctive hyperbaric oxygen in cervical necrotizing fasciitis. Otolaryngol Head Neck Surg. 2009;140(5):730-734.

13. Lingaraj JB, Rao S, Kotrashetti SM, et al. Necrotizing cervical fasciitis: a case report and review of literature. J Maxillofac Oral Surg. 2010;9(1):54-56.

14. Huang D, Deng W, Li C, et al. Craniocervical necrotizing fasciitis of odontogenic origin with thoracic extension. J Craniofac Surg. 2011;22(3):1109-1111.

15. Sandner A, Moritz S, Unverzagt S, et al. Cervical Necrotizing Fasciitis--The Value of the Laboratory Risk Indicator for Necrotizing Fasciitis Score as an Indicative Parameter. J Oral Maxillofac Surg. 2015;73(12):2319-2333.

16. Muhammad JK, Almadani H, Al Hashemi BA, et al. The value of early intervention and a multidisciplinary approach in the management of necrotizing fasciitis of the neck and anterior mediastinum of odontogenic origin. J Oral Maxillofac Surg. 2015;73(5):918-927.

17. Sarna T, Sengupta T, Miloro M, et al. Cervical necrotizing fasciitis with descending mediastinitis: literature review and case report. J Oral Maxillofac Surg. 2012;70(6):1342-1350.
18. Zhang WJ, Cai XY, Yang C, et al. Cervical necrotizing fasciitis due to methicillin-resistant Staphylococcus aureus: a case report. Int J Oral Maxillofac Surg. 2010;39(8):830-834.

19. Sangamesh NC, Vidya KC, Roopa GS, et al. Necrotizing fasciitis of odontogenic origin in a nonimmunocompromised patient: a rare case report. Journal of the Scientific Society. 2014;41(3):179-182.

20. Shand JM, Breidahl A, Hing NR, et al. Ascending necrotising fasciitis as a result of odontogenic infection: a report of two cases. Aust Dent J. 2001;46(2):134-138.

21. Umeda M, Minamikawa T, Komatsubara H, et al. Necrotizing fasciitis caused by dental infection: a retrospective analysis of 9 cases and a review of the literature. Oral Surg Oral Med Oral Pathol Oral Radiol Endod. 2003;95(3):283-290.

22. Gausepohl JS, Wagner JG. Survival from cervical necrotizing fasciitis. West J Emerg Med. 2015;16(1):172-174.

23. Chueng K, Clinkard DJ, Enepekides D, et al. An Unusual Presentation of Ludwig's Angina Complicated by Cervical Necrotizing Fasciitis: A Case Report and Review of the Literature. Case Rep Otolaryngol. 2012: 931350.

24. Cottom H, Gallagher JR, Dhariwal DK, et al. Odontogenic cervico-fascial infections: a continuing threat. J Ir Dent Assoc. 2013; 59(6):301-307.

25. Quereshy FA, Baskin J, Barbu AM, et al. Report of a case of cervicothoracic necrotizing fasciitis along with a current review of reported cases," J Oral Maxillofac Surg. 2009;67(2):419-423.

26. Chrcanovic BR, Custódio ALN. Cervical necrotizing fasciitis of odontogenic origin: case report. Serbian Dental Journal. 2009;56:194-200.

27. Eades R. Radiology in practice: a case study. Prim Dent J. 2(1):40-45. 